Cita: Raimundi, M. J.; Sarries, S.; Corti, J. F.; \& Celsi, I. (2021). La percepción de entrenadores de voleibol y básquetbol de formación acerca de los contenidos de las charlas precompetitivas y de entretiempo. Cuadernos de Psicología del Deporte, 21(3), 139-155

\title{
La percepción de entrenadores de voleibol y básquetbol de formación acerca de los contenidos de las charlas precompetitivas y de entretiempo
}

\section{The perception of youth volleyball and basketball coaches of contents of precompetitive and halftime talks}

\section{A percepção dos treinadores de treinadores de vôlei e basquete sobre os conteúdos das palestras pré-competitivas e de meio período}

\author{
Raimundi, María Julia; Sarries, Sofía²; Corti, Juan Facundo ${ }^{3}$; Celsi, Ignacio ${ }^{4}$ \\ ${ }^{1}$ Instituto de Psicología Básica, Aplicada y Tecnología (IPSIBAT-CONICET) - Universidad Abierta \\ Interamericana, Argentina \\ ${ }^{2}$ Facultad de Psicología, Universidad Católica Argentina, Argentina \\ ${ }^{3}$ Facultad de Psicología, Universidad de Buenos Aires, Argentina \\ ${ }^{4}$ CONICET - Facultad de Psicología, Universidad de Buenos Aires, Argentina
}

\begin{abstract}
RESUMEN
El objetivo del presente trabajo fue estudiar la percepción de entrenadores sobre los contenidos de charlas precompetitivas y de entretiempo desde la estrategia metodológica de los métodos mixtos. Participaron 21 entrenadores de básquetbol y voleibol, en su mayoría varones $(\mathrm{n}=20)$, de categorías formativas ( $\mathrm{u} 15$, u17 y u19) de Buenos Aires (Argentina). Se realizaron entrevistas individuales y semiestructuradas a los entrenadores y a través de la Teoría Fundamentada, se realizó un análisis cualitativo, mediante el uso del programa Atlas.Ti 7.5. Los entrenadores destacaron cinco aspectos relevantes respecto de los contenidos durante las charlas previas y de entretiempo: aspectos estratégicos, psicológicos, comunicacionales, variables que influyen en la elección de los contenidos y contenidos específicos de entretiempos. A través del análisis de los documentos primarios, se asociaron las citas significativas a cada una de las categorías. La categoría mayormente mencionada (i.e. más fundamentada en los datos) fue "Aspectos estratégicos", mientras que los "Aspectos psicológicos" estuvieron en segundo lugar. Se realizó un análisis de asociación para estudiar la prevalencia de las categorías emergidas del análisis en función del deporte, la rama y la categoría dirigida. Los resultados de este estudio aportan claridad respecto a cuáles son los contenidos valorados por entrenadores en estos momentos específicos de interacción y qué estrategias utilizan para comunicarlos. Tener en cuenta esta información será de capital importancia para desarrollar estrategias psicológicas significativas que potencien los recursos de los entrenadores para hacer frente a las demandas en estas situaciones específicas.
\end{abstract}

Palabras clave: Autopercepciones, Conducta verbal, Competiciones, Entrenador, Deporte Adolescente.

\section{ABSTRACT}

The present study aims to explore the perception of coaches on the contents of pre-competition and halftime talks using a mixed-methods approach. Participants were 21 youth basketball and volleyball coaches (u15, u17 and u19), 


\section{Raimundi, M. J.; Sarries, S.; Corti, J. F.; Celsi, I.}

mostly males $(n=20)$, from Buenos Aires (Argentina). Individual and semi-structured interviews were conducted to the coaches and through Grounded Theory the information collected was subjected to a qualitative analysis with program Atlas.Ti 7.5. Coaches highlight five relevant aspects of the contents in pre-competition and halftime talks: strategic, psychological, communicational, variables that influence the choice of content and specific halftime content. Analyzing the primary documents, significant quotes were associated with each category. The most mentioned category (i.e. greater frequency in data) was 'Strategic aspects', while 'Psychological aspects' were placed secondly. An association analysis was carried out to study the prevalence of the categories emerged from the analysis based on the sport, branch, and category. The results of this study provide clarity regarding which are the most valued contents for coaches to spread in these specific moments of interaction and which strategies they use to communicate them. Taking this information into account will be crucial to develop meaningful psychological strategies that enhance coaches' resources to cope with the demands of these specific situations.

Keywords: Self-perceptions, Verbal Behavior, Competitions, Coach, Youth Sport.

\section{RESUMO}

O presente estudo tem como objetivo explorar a percepção dos treinadores sobre o conteúdo das palestras antes da competição e no intervalo da estratégia de métodos mistos. Participaram 21 treinadores de basquete e vôlei, a maioria homens ( $\mathrm{n}=20)$, das categorias juvenil (u15, u17 e u19) de Buenos Aires (Argentina). Entrevistas individuais e semiestruturadas foram conduzidas aos treinadores e, por meio da Teoria Fundamentada, as informações coletadas foram submetidas a uma análise qualitativa com o programa Atlas.Ti 7.5. Os treinadores enfatizam cinco aspectos relevantes em relação ao conteúdo durante as palestras antes da competição e no intervalo: estratégicas, psicológicas, comunicacionais, variáveis que influenciam a escolha do conteúdo e o conteúdo específico no intervalo. Por meio da análise dos documentos primários, citações significativas foram associadas a cada categoria. A categoria mais mencionada (i.e. mais informada pelos dados) foi "Aspectos estratégicos", ficando em segundo lugar os "Aspectos psicológicos". Foi realizada uma análise de associação para estudar a prevalência das categorias emergidas da análise com base no esporte, no ramo e na categoria-alvo. Os resultados deste estudo fornecem clareza sobre quais são os conteúdos valorizados pelos treinadores nesses momentos específicos de interação e quais estratégias eles usam para comunicá-los. Levar essas informações em consideração será de suma importância para o desenvolvimento de estratégias psicológicas significativas que aprimorem os recursos dos treinadores para lidar com as demandas nessas situações específicas.

Palavras chave: Auto-percepções, Conduta Verbal, Competições, Treinador, Esporte adolescente.

\section{INTRODUCCIÓN}

El papel de entrenadores y entrenadoras (en adelante entrenadores) es objeto de estudio fundamental en las Ciencias del Deporte, y específicamente para las disciplinas interesadas en el deporte formativo, ya que, a través de sus conductas, los entrenadores pueden impactar no sólo en el funcionamiento deportivo sino también en el bienestar de los deportistas (Adie et al., 2012; Balaguer et al., 2008). Por lo tanto, para la Psicología del deporte se ha considerado relevante realizar un seguimiento de aquellas aptitudes, actitudes y valores que orientan la conducta del entrenador y que no sólo se ven influenciadas por el mismo profesional sino también por el contexto en el cual desempeñan su tarea (Castillo, Adell et al., 2018; Marcelo y Gallego-Domínguez, 2018).

Entre las diversas conductas llevadas a cabo por los entrenadores, la conducta verbal es de capital importancia para organizar y brindar información a los deportistas durante entrenamientos y competencias (Lawrence y Kingston, 2007; More y Franks, 2004). La conducta verbal se define como el contenido del mensaje que emite el entrenador, sus indicaciones durante el desarrollo de la competencia o las advertencias que da a los jugadores cada vez que se pide tiempo y se suspende el juego (Anguera y BlancoVillaseñor, 2003). El feedback que dan los entrenadores es un componente esencial de esta conducta verbal, dado que es utilizado para orientar, corregir, premiar o castigar a los deportistas y se ha demostrado su importancia como predictor de la experiencia deportiva en deportistas en etapas de formación (e.g. Curran et al., 2015; Luque et al., 2019).

Considerando a la metodología observacional como un método mixto en sí mismo, que permite la recolección de datos directamente de los participantes en el contexto deportivo (Anguera y Hernández- 


\section{Percepciones de entrenadores sobre charlas precompetitivas y entretiempos}

Mendo, 2013), se contempla a la conducta verbal como un comportamiento capaz de ser percibido y objetivado. Diferentes trabajos han estudiado los comportamientos de los entrenadores durante entrenamientos y competiciones con el objetivo de comprender su impacto de estas conductas en las experiencias de los deportistas (e.g. Guzmán-Luján et al., 2014; N. Smith et al., 2016). Marques et al. (2015) han encontrado relaciones entre los estilos de comunicación de los entrenadores y el clima motivacional percibido por sus jugadores, destacándose que las conductas de apoyo y las instrucciones de los entrenadores se asocian a la percepción de un clima de implicación a la tarea, mientras que las percepciones de comportamientos punitivos del entrenador se asocian a climas de implicación en el ego y conflictos intra-equipo. Asimismo, algunos estudios han examinado cómo se desarrolla y varía la conducta verbal de los entrenadores ante distintos factores situacionales de la competencia, como las acciones de juego (Guzmán Luján y Calpe-Gómez, 2012), el marcador del partido (Calpe-Gómez et al., 2013), los momentos de pausa (i.e. charlas previas, de entretiempo y tiempos muertos) (Lorenzo et al., 2013).

En este sentido, la gran cantidad de literatura sobre la observación de los entrenadores ha encontrado sistemáticamente que la conducta verbal representa uno de los comportamientos más comunes estudiados (Mason et al., 2020). Sin embargo, un área que ha recibido menos atención es la investigación sobre las autopercepciones y creencias de los entrenadores acerca de la conducta verbal que utilizan con sus deportistas (Smith y Cushion, 2006). En un reciente estudio cualitativo con entrenadores de élite (Mason et al., 2020) encontraron que estos ven al feedback como una herramienta para mejorar el rendimiento, desarrollar la confianza de los deportistas, ayudarlos a monitorear el progreso y como una herramienta para mejorar su propio rendimiento. Estos autores plantean que este tipo de estudios pueden proporcionar más detalles acerca de los contextos y limitaciones que los entrenadores tienen en su trabajo diario. Asimismo, Balagué Serré et al. (2014) muestran que los entrenadores suelen percibir su rol como una tarea compleja que demanda desarrollar y gestionar un conjunto de habilidades y conocimientos con las cuales poder guiar y dirigir a su equipo. En este sentido, la autopercepción de competencias del rol de los entrenadores se convierte en una importante herramienta en la determinación de los conocimientos y aptitudes necesarias para el desarrollo óptimo y eficaz de tan compleja tarea.

$\mathrm{Al}$ igual que otros deportes de conjunto, el reglamento del básquetbol y el voleibol permite la comunicación y el diálogo entre entrenador y jugadores bajo ciertos momentos de la competencia, como en las charlas previas y en los entretiempos (en básquetbol) y entre los sets (en voleibol). Esto le da la posibilidad no sólo de detener el ritmo del partido sino también de organizar a sus jugadores, brindándoles instrucciones y estrategias de manera próxima e inmediata. En este lapso, el entrenador puede transmitir determinada información relevante a su equipo y la eficacia de sus intervenciones estará altamente determinada por los recursos y estrategias que elija emplear. Dada la importancia del papel de los entrenadores en el rendimiento y bienestar de los deportistas, especialmente en los más jóvenes, es de interés conocer los contenidos que consideran relevantes incluir en las charlas durante los partidos, desde su propia perspectiva.

Por lo tanto, el objetivo del presente trabajo es estudiar la percepción de los entrenadores acerca de los contenidos de las charlas previas y de entretiempo durante partidos de voleibol y básquetbol de categorías formativas. El interés del estudio reside en conocer cuáles son los aspectos que los entrenadores consideran relevantes a la hora de realizar estas acciones durante la competencia. Ello permitirá profundizar el conocimiento de las estrategias utilizadas por los entrenadores para el diseño de intervenciones con el fin de optimizar sus recursos y herramientas como comunicadores y líderes de sus equipos.

\section{MATERIAL Y MÉTODOS}

Tipo de estudio y diseño

Estudio de estrategia descriptiva (Ato et al., 2013) con utilización de los métodos mixtos como estrategia metodológica, integrando la vertiente cualitativa y cuantitativa con el mismo fin y dentro del mismo estudio (Anguera y Mendo, 2016; Morales Sánchez et al., 2020). Considerando que el presente estudio tiene por objeto de interés lo expresado por sus protagonistas, se utilizó, en primer lugar la metodología cualitativa en tanto no se busca la representatividad de los datos sino la posibilidad de profundizar el sentido de los fenómenos sociales (Maxwell, 2012) y brinda la oportunidad de indagar 


\section{Raimundi, M. J.; Sarries, S.; Corti, J. F.; Celsi, I.}

los significados subjetivos y las concepciones desde los propios sujetos protagonistas, dado que ellos determinan en cierto grado su comportamiento (Schmidt et al., 2008). En segundo lugar, se realizó un tratamiento cuantitativo, permitiendo un análisis de datos más robusto tomando las ventajas que brinda cada enfoque (Anguera y Mendo, 2016).

\section{Participantes}

La muestra fue seleccionada de manera intencional buscando que los participantes proporcionen un sentido de comprensión profunda del ambiente y el problema de investigación (Creswell, 2013; Hernández-Sampieri y Mendoza Torres, 2018) y en base a la disponibilidad, accesibilidad y voluntad propia de participar en el estudio (Cajina-Guedeat y Reyes-Bossio, 2021; Mason et al., 2020). Desde la estrategia cualitativa se la puede considerar como una muestra típica o intensiva, dado que se eligen casos de un perfil similar, pero que se consideran representativos de un segmento de la población, una comunidad o una cultura (i.e. no en un sentido estadístico, sino de prototipo). En el presente estudio se consideraron dos deportes (i.e. voleibol y básquetbol) dado que en Argentina son deportes con un desarrollo similar en cuanto estructura deportiva, cantidad de deportistas federados y propuestas de formación de entrenadores. Particularmente, para poder ejercer como entrenador en estos deportes es requisito realizar el curso de entrenador habilitante otorgado por las respectivas federaciones deportivas. Asimismo, respecto del problema de investigación, en estos deportes la estructura de los partidos es similar, teniendo momentos de preparación (i.e. charla precompetitiva y detención del juego -entretiempos y entre sets-) equiparables.

Participaron 21 entrenadores de básquetbol y voleibol que dirigen equipos de categorías formativas (u15, u17 y u19) en las respectivas federaciones deportivas del Área Metropolitana de Buenos Aires (Argentina). La distribución de los participantes en función del deporte y la rama se presenta en la Tabla 1. Respecto del género, casi la totalidad de los participantes fueron entrenadores de género masculino $(n=20)$ a excepción de una entrenadora $(n=1)$. La edad media de los participantes fue de 35.5 años $(D T=8.9)$, y todos ellos realizaron al menos dos niveles de los cursos nacionales de entrenador (siendo cuatro en total en el voleibol y tres en el básquetbol). Entre los participantes, 20 entrenadores $(95.2 \%)$ tenían un trabajo adicional al momento de la recolección, la mitad de los cuales se desarrollaban en rubros no relacionados a la actividad física. La mayoría de los entrenadores $(71.4 \%)$ refirieron dedicarse a tiempo parcial a su labor de entrenador. Todos los entrenadores entrevistados fueron jugadores de sus respectivos deportes, y 13 de ellos (61.9\%) continúan su práctica en la actualidad.

Tabla 1

Características de los entrenadores en función del deporte y la rama dirigida

\begin{tabular}{cccc}
\hline & Básquetbol & Voleibol & Total \\
& $n(\%)$ & $n(\%)$ & $n(\%)$ \\
\hline Rama masculina & $10(83.3)$ & $4(44.4)$ & $14(66.7)$ \\
Rama femenina & $2(16.7)$ & $5(55.6)$ & $7(33.3)$ \\
\hline Total & $12(57.1)$ & $9(42.9)$ & $21(100)$ \\
\hline
\end{tabular}

\section{Instrumentos}

Entrevistas individuales. Se realizaron entrevistas semiestructuradas que tuvieron una duración aproximada de 10 minutos. Se indagó sobre las percepciones de los entrenadores acerca de los contenidos de las charlas previas y de entretiempo en los partidos. La guía para la entrevista fue desarrollada por el equipo de investigación (con miembros especialistas en evaluación psicológica y practicantes de las disciplinas deportivas). A su vez, se realizó una prueba piloto con dos entrenadores de los mismos deportes que participaron. Las preguntas-guía finales fueron: ¿qué contenidos suelen tener las charlas previas a los partidos y las del entretiempo? ¿qué es lo que se quiere transmitir a los/as jugadores/as?

\section{Procedimiento}

Este proyecto cuenta con el aval del Comité de Ética de la Facultad de Psicología de la Universidad de Buenos Aires y forma parte de una investigación más amplia que se orienta al estudio de las percepciones de entrenadores y deportistas de categorías formativas acerca de las conductas que llevan a cabo en sus entrenamientos y partidos. Se contactó a las autoridades de los clubes y a los entrenadores invitándolos a participar del estudio y se les explicó el propósito del proyecto. La participación de los entrenadores fue voluntaria y confidencial, previo consentimiento escrito. Las entrevistas se realizaron telefónicamente y fueron grabadas. 


\section{Percepciones de entrenadores sobre charlas precompetitivas y entretiempos}

\section{Análisis de datos}

Considerando el objetivo del presente estudio y de acuerdo con los lineamientos de los métodos mixtos, basados en la ordenada sucesión de etapas que dan cuenta de la complementariedad cualitativacuantitativa (Anguera y Hernández-Mendo, 2016; Morales-Sánchez et al., 2020), se comenzó la obtención de datos utilizando el enfoque cualitativo. Se utilizó el procedimiento de análisis de la Teoría Fundamentada (Strauss y Corbin, 1990, 1997), que implica que los hallazgos van emergiendo fundamentados en los datos recolectados, lo cual permite el desarrollo de un conjunto de proposiciones teóricas. Se realizó un análisis de contenido temático a las respuestas de los entrenadores, siguiendo los lineamientos propuestos por Strauss y Corbin (1990). En primer lugar, uno de los miembros del equipo realizó la transcripción de las grabaciones de las entrevistas y otro miembro realizó una validación de éstas. Para analizar su contenido se utilizó el programa Atlas.Ti 7.5 y se organizaron las entrevistas en un único archivo (Unidad Hermenéutica; UH), en el que se almacenó toda la información producida durante el análisis. Dos investigadores trabajaron de manera independiente en duplicados del archivo e identificaron las unidades de significado relevantes de tipo "libre flujo" (Hernández-Sampieri y Mendoza Torres, 2018) para el objetivo de investigación, asignándoles un código (codificación abierta o de primer nivel). Luego se compararon las unidades de significado y la descripción de las categorías realizada por cada investigador y se estableció un libro de códigos a partir de las categorías que, a juicio de ambos investigadores, cumplían con los criterios de fácil inferencia y aplicación y de relevancia, garantizando de este modo la concordancia consensuada (Anguera, 1990; Lapresa Ajamil et al., 2021).

Como criterio de validez en metodología cualitativa, un investigador externo al proceso realizado revisó y auditó las categorizaciones elaboradas. Este procedimiento se orientó a establecer la credibilidad, dependencia y confirmabilidad de los datos analizados previamente (Hernández-Sampieri y Mendoza Torres, 2018). Por último, dos investigadores realizaron una lectura del material utilizando las reglas explícitas desarrolladas para codificar las unidades de significado en diferentes $\mathrm{UH}$, que posteriormente fueron fusionadas para obtener una UH definitiva (Figura 1). Como control de la calidad del dato, el cálculo del coeficiente Kappa (Cohen, 1960) fue implementado para evaluar la fuerza de la concordancia entre ambos observadores, obteniéndose un coeficiente casi perfecto $(\kappa=.83)$ (Landis y Koch, 1977).

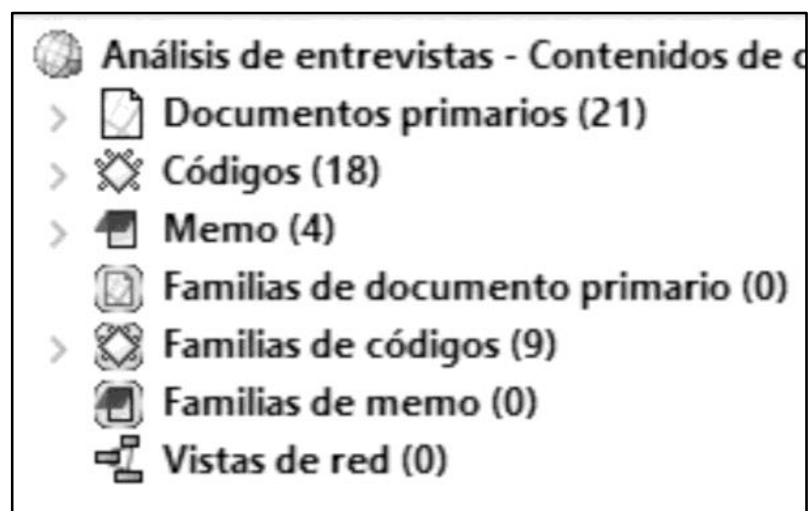

Figura 1. Descriptivos del análisis del contenido en la Unidad Hermenéutica definitiva

En la UH definitiva, los documentos primarios corresponden a cada una de las entrevistas realizadas. Las citas son las segmentaciones de los documentos primarios que fueron consideradas significativas y que pueden ser asociadas a un código. Los códigos son los elementos resultantes del análisis y se conformaron como un sistema mixto que combinó formatos de campo y un sistema de categorías (Anguera et al., 2011). Las familias de códigos son categorías centrales o de orden superior, que permiten agrupar códigos en base a un criterio determinado. Por su parte, los memos son todas aquellas anotaciones que los investigadores han considerado importante recoger o aclarar para la realización de todo el análisis (PérezLópez et al., 2015).

Para el tratamiento cuantitativo de la información, se exportó la base de datos al programa SPSS v.23 y se realizó un conteo de las frecuencias de las categorías de primer nivel y centrales que fueron informadas por los entrenadores. Para ello, se estimó el porcentaje de participantes que mencionó la categoría, por lo menos, una vez. Asimismo, para profundizar la comprensión del objetivo del presente estudio, se realizó un análisis de asociación (Chi cuadrado) en función del deporte, la rama y la categoría dirigida, considerando la realización de este tratamiento cuantitativo desde técnicas analíticas adecuadas para datos categóricos (Anguera y Hernández-Mendo, 2016). 


\section{Raimundi, M. J.; Sarries, S.; Corti, J. F.; Celsi, I.}

\section{RESULTADOS}

En la Tabla 2 se presenta un resumen de las categorías de primer nivel (códigos) y las categorías centrales (familias de códigos) de las charlas previas y de entretiempo, con ejemplos de fragmentos de las entrevistas de los entrenadores. A continuación, se describen las categorías propuestas y los criterios definidos para la inclusión de elementos en cada una de ellas.

Tabla 2

Categorías centrales (familias de códigos), de primer nivel (códigos) y ejemplos

\begin{tabular}{|c|c|c|}
\hline $\begin{array}{c}\text { Categoría } \\
\text { central }\end{array}$ & $\begin{array}{l}\text { Categoría de } \\
\text { primer nivel }\end{array}$ & Ejemplo \\
\hline $\begin{array}{l}\text { I. Aspectos } \\
\text { estratégicos }\end{array}$ & $\begin{array}{l}\text { a. Plan de juego } \\
\text { b. Información del rival } \\
\text { (scouting) }\end{array}$ & $\begin{array}{l}\text { "En las charlas previas a los partidos tratamos de enfocar el plan que haremos } \\
\text { (...) y algo que hayamos trabajado en la semana" (BF, u19). } \\
\text { "Previo al partido yo siempre charlo sobre contra quién vamos a jugar, qué tienen, } \\
\text { cómo nos jugarían ellos, en teoría" (VM, u15-u17). }\end{array}$ \\
\hline $\begin{array}{l}\text { II. Aspectos } \\
\text { psicológicos }\end{array}$ & $\begin{array}{l}\text { a. Regulación de la } \\
\text { activación } \\
\text { b. Filosofía de } \\
\text { afrontamiento de la } \\
\text { competencia } \\
\text { c. Apoyo a la autonomía } \\
\text { d. Identidad de equipo / } \\
\text { Comunicación grupal }\end{array}$ & $\begin{array}{l}\text { "Ser puntual, muchas veces para tranquilizar al equipo, y otras para darle un } \\
\text { poquito más de envión anímico" (VF, u17 y u19). } \\
\text { "Por último, un refuerzo de la filosofía, de todo el esfuerzo que venimos haciendo } \\
\text { para... para... para llegar al momento de la competencia en la que estamos o en } \\
\text { el inicio del torneo. Y siempre digo lo mismo... que disfruten... que disfruten del } \\
\text { juego" (BM, u19). } \\
\text { "En temporada regular les pido que tomen lectura del juego por ellos mismos, } \\
\text { bajo la consigna "cuanto menos necesiten del entrenador, mejores jugadores van } \\
\text { a ser"” (BM, u17-19). } \\
\text { "Se habla mucho primero de lo que tenemos que trabajar de la } \\
\text { red para nuestro lado... La identidad de nuestro equipo" (VF, u17-u19). }\end{array}$ \\
\hline $\begin{array}{l}\text { III. Aspectos } \\
\text { comunicacion } \\
\text { ales }\end{array}$ & $\begin{array}{l}\text { a. Características del } \\
\text { mensaje } \\
\text { b. Uso de recursos físicos } \\
\text { / audiovisuales }\end{array}$ & $\begin{array}{l}\text { "Yo creo que en los tiempos siempre son dos o tres cosas nada más, porque si } \\
\text { tiras mucha información no sirve. Ser puntual" (VF, u17 y u19). } \\
\text { "Si tenemos la posibilidad de tener un pizarrón, yo ya previo a la charla marqué } \\
\text { algunas cosas en el pizarrón para tener un hilo en la charla" (BM, u19). }\end{array}$ \\
\hline $\begin{array}{l}\text { IV. Variables } \\
\text { que influyen } \\
\text { en la elección } \\
\text { de contenidos }\end{array}$ & $\begin{array}{l}\text { a. Nivel de competencia } \\
\text { b. Encuadre }\end{array}$ & $\begin{array}{l}\text { "[La charla] es depende el nivel de competencia" (BF, u15). } \\
\text { "[La charla] previa depende el partido, el momento del año y la situación del } \\
\text { equipo" (BM, u17-u19). }\end{array}$ \\
\hline $\begin{array}{l}\text { V. } \\
\text { Entretiempo }\end{array}$ & $\begin{array}{l}\text { a. Contenidos específicos } \\
\text { del entretiempo }\end{array}$ & $\begin{array}{l}\text { "En el entretiempo tratamos de evaluar si estamos cumpliendo con los objetivos } \\
\text { trazados. Si el plan del juego se está llevando adelante de acuerdo a lo establecido } \\
\text { y en caso de que no, buscar las razones y tratar de buscar la solución para poder } \\
\text { cumplirlo" (BF, u17). }\end{array}$ \\
\hline
\end{tabular}

Nota $. \mathrm{B}=$ básquetbol, $\mathrm{V}=$ voleibol, $\mathrm{F}=$ femenino, $\mathrm{M}=$ masculino

\section{Aspectos estratégicos}

Esta categoría hace referencia a la información técnica y táctica que brindan los entrenadores sobre el propio equipo o sobre el equipo rival, con la finalidad de facilitar una ventaja estratégica durante los partidos.

\section{I. a. Plan de juego (información propia)}

Aspectos técnico-tácticos del propio equipo. Dar información para ejecutar, para resolver las situaciones de juego y para afrontar las características del otro equipo. Refuerzo de lo que se entrenó entre competiciones.
"En las charlas previas, trato de recordar lo que venimos entrenando en las semanas anteriores" (voleibol femenino, u15).

En basquetbol se hace un especial énfasis en las cuestiones defensivas, aunque también se proponen algunas cuestiones ofensivas (sistemas de juego, puntos fuertes del propio equipo).

Las charlas previas son la organización y tratar de plasmar en el partido lo que entrenamos en la semana. Generalmente trato de buscar tres claves de juego para poder cumplirlo en el partido. Luego las reglas defensivas que debemos cumplir en el partido de acuerdo a lo entrenado (...) y por último 


\section{Percepciones de entrenadores sobre charlas precompetitivas y entretiempos}

las reglas ofensivas de ese partido. (Basquetbol femenino, u17).

"En relación a las charlas previas, yo lo que intento es terminar de pasarles los tres o cuatro argumentos defensivos, (...) que lo que estuvimos haciendo tiene coherencia" (basquetbol masculino, u19)

\section{I. b. Información del rival (scouting)}

Brindar información sobre el equipo al que se va a enfrentar: sus puntos fuertes, sus tendencias en el juego, muchas veces apoyado en análisis previos con videos o estadísticas obligatorias en ciertos niveles competitivos (i.e. scouting).

"En los partidos, yo siempre tiro tres o cuatro cosas del otro equipo, de lo que hace" (voleibol femenino, u17 y u19).

"Con las chicas jugamos la liga, y la liga te permite mirar y [hacer] scouting. Le mostrás aspectos individuales, las jugadas del rival" (basquetbol masculino, u15).

\section{Aspectos psicológicos}

Acciones que buscan un efecto directo en el área psicosocial de los deportistas entrenados, o condiciones generales del modo de llevar adelante la actividad que impactan a largo plazo en aspectos psicológicos de los atletas.

\section{II. a. Regulación de la activación}

Remite a la energía previa al partido. Comúnmente los entrenadores pueden mencionarla como "motivación", pero refiere a aspectos más vinculados a cantidad de energía y su regulación, y a una actitud positiva para afrontar la competencia.

"Y siempre un golpe motivacional para activarlos" (voleibol masculino, u15 y u17).

"Luego sigue una parte emocional, depende el momento del equipo que se trate" (basquetbol masculino, u17 y u19).

\section{II. b. Filosofía de afrontamiento de la competencia}

Se trata de un encuadre global para el afrontamiento de los partidos en donde se enfatiza el aprendizaje, el disfrute y la valoración del propio trabajo a partir de la mejora y la sensación de competencia centrada en la tarea, es decir, enfocada en el juego y no en el resultado.

"Sobre todo quiero hacer entender a los chicos que el partido es un espacio para aprender, para mejorar, haciendo referencia a aprender fundamentos, cuestiones técnicas, tácticas, estratégicas y a competir" (basquetbol masculino, u15).

Hacerles entender que desde el minuto cero, desde la entrada en calor tenemos que estar metidos y concentrados en la tarea. (...) Entender que lo más importante que podemos hacer, lo más inteligente es jugar el juego, independientemente de si está saliendo bien o no. (Basquetbol masculino, u19).

\section{II. c. Apoyo a la autonomía}

Los entrenadores intentan acompañar, dar confianza y apoyo a sus jugadores a través de la participación y la autonomía para tomar decisiones.

"Tratar de trasladarles confianza. (...) Y lo más importante que creo, que trato de que se genere, es que sientan una especie de... un escenario de seguridad" (basquetbol masculino, u19).

"Más o menos trato de que sea algo participativo, no bajar una línea de dirección como si fuera un monólogo" (voleibol femenino, u15 y u17).

\section{II. d. Identidad del equipo / comunicación grupal}

Refiere a los aspectos que hacen a la cohesión y disfrute del equipo desde la comunicación y la identidad.

"Además de favorecer la charla entre ellos" (basquetbol masculino, u17 y u19).

"Que disfruten del compañero" (basquetbol masculino, u19).

\section{Aspectos comunicacionales}

Esta categoría central engloba aquellas consideraciones y estrategias que los entrenadores tienen en cuenta al momento de comunicarse con sus deportistas.

\section{III. a. Características del mensaje}

Refiere a la cantidad, extensión y estructura de la información a transmitir: poca información, puntual, sencilla, utilizando el "método / intervención sándwich" o usando el humor debido a la brevedad del tiempo disponible, tanto antes de los partidos como en el entretiempo o entre sets.

"Entre set y set lo que hago generalmente es poner un punto positivo, después un punto a mejorar y después cerrar con otro punto positivo" (voleibol masculino, u15 y u17).

"Trato siempre de que tenga algo de humor, boludez [sic], algún chiste, siempre trato de divertirnos. Mucho contenido no" (basquetbol masculino, u15 y u17). 


\section{Raimundi, M. J.; Sarries, S.; Corti, J. F.; Celsi, I.}

También hace referencia a los destinatarios: a veces es a todo el grupo y a veces algunos jugadores puntuales. "Charlas grupales e individuales" (basquetbol masculino, u17 y u19).

\section{III. b. Uso de recursos físicos / audiovisuales}

Utilización de vídeos como recurso para activar o generar algún estado emocional particular previo a un partido o el uso de un soporte didáctico, como una pizarra, para ordenar la información a transmitir.

Hasta que pusimos un video motivacional, con De Niro, hicimos parte del juego, parte de nosotros con Al Pacino hablándole como en 'Un domingo cualquiera', eso es una filmación que le pasamos a los jugadores. (...) Esas son todas herramientas que hay que utilizar, pero en un momento determinado, no siempre. También a veces a mí me quedan dudas si eso es bueno o malo, porque habló el nene, el hijito que tiene cuatro años: 'papá', y el tipo está concentrado para meterse y se le aflojó toda la estructura, toda la estructura de guerrero por decirlo de alguna manera, lo pusiste tierno en un momento en que no tiene que estar tierno, por eso tengo dudas si eso sirvió o no sirvió, pero si lo hicimos. (Básquetbol masculino, u19).

\section{Variables que influyen en la elección de los contenidos}

Se trata de las condiciones contextuales que inciden en la decisión del entrenador respecto a qué incluir en las charlas técnicas.

\section{IV. a. Nivel de la competencia}

Muchas veces el contenido de la charla previa al partido puede variar considerando el nivel competitivo del equipo. Cuanto mayor es el nivel, más estratégico y específico se vuelve el contenido. Entrenadores de niveles formativos no suelen brindar tanta información estratégica (del rival o del propio plan de juego).

"Cuando estás hablando a otro nivel, ya hacés scout, en mi caso en la Liga de Desarrollo, cuáles eran las jugadas que más hacía el equipo contrario, los porcentajes de cada uno de los jugadores del equipo contrario" (basquetbol masculino, u19).

"La mayoría de las veces tengo que estar alerta a charlas sobre autoconfianza, motivación, autocrítica... Muy pocas veces son técnicas-tácticas" (basquetbol masculino, u15 y u17).

\section{IV. b. Encuadre}

Refiere al momento de la temporada, a la importancia del partido o a la situación actual del equipo.

Los primeros minutos son del momento del año en el que nos encontramos: en el inicio, en el medio o en el cierre del año. Tiene que ver con la competencia en la que estamos participando y en el momento de la competencia. Si bien yo estoy con chicos en formación... Eh... Con chicos juveniles y u17 y u19... Eh...también con el momento, a ver en qué momento de la competencia si es una semifinal o una final eso es el principio de la charla. (Basquetbol masculino, u19).

\section{Contenidos específicos del entretiempo}

Las categorías desarrolladas (I a IV) aplican a la descripción de las percepciones de los entrenadores tanto en charlas previas como en entretiempos. Sin embargo, los entrenadores enfatizan algunas diferencias entre las charlas previas y los entretiempos. Por ello, en el análisis emerge esta categoría referida específicamente a este momento de la competencia. En los entretiempos se dan ajustes, correcciones y redireccionamientos de lo planteado en la charla previa al partido (desde lo estratégico del plan de juego y del análisis del rival) en función de cómo se esté dando el partido hasta el momento. Se aplican los mismos aspectos comunicacionales de las charlas previas.

Y durante los entretiempos, que sería durante los sets, creo que siempre es remarcar la táctica que se puede usar, corregirla en el caso que haya sido la táctica equivocada, modificar algún planteo que hayamos preparado durante la semana y no lo estemos llevando bien a cabo. (Voleibol masculino, u19).

"En el entretiempo lo que se suele hacer es reforzar ciertas cosas, qué seguir haciendo, qué no seguir haciendo, a quién marcar, a quien darle la pelota en ataque y demás" (basquetbol masculino, u15).

"[En el entretiempo se dan] correcciones tácticas, charlas grupales e individuales. Detalles a pulir para lo que queda del partido. Refuerzo positivo a las cosas que vienen bien" (basquetbol masculino, u17 y u19). 


\section{Percepciones de entrenadores sobre charlas precompetitivas y entretiempos}

\section{Prevalencia de las categorías centrales y de primer nivel}

A través del análisis de los documentos primarios, se asociaron las citas significativas a cada uno de los códigos (categorías de primer nivel). La Figura 2 presenta la frecuencia (fundamentación) en la que cada uno de estos códigos fue asociado a una cita en los documentos primarios (entrevistas) y su familia correspondiente.

El código "Plan de juego" obtuvo la mayor cantidad de fundamentaciones $(n=29)$, seguido por "Información del rival (scouting)" $(n=17)$, mientras que "Identidad de equipo / Comunicación grupal" fue el menos codificado $(n=3)$. Los códigos vinculados al deporte ("Básquetbol" y "Voleibol"), la rama ("Rama Masculina" y "Rama Femenina") y la categoría ("u15", “u17" y "u19") fueron códigos "descriptores" que se utilizaron para caracterizar a los participantes. En la Tabla 3 se presenta la frecuencia de entrenadores que mencionaron alguna de las categorías en función de los códigos descriptores (deporte, rama y categoría dirigida).

La asociación (prueba $\chi 2$ ) entre la prevalencia de las categorías en función del deporte, la rama dirigida y la categoría mostró que no hay una relación significativa en función de la rama y categoría dirigida (u15, u17 y u19) para ninguna de las categorías del análisis, mientras que se encontró una asociación significativa que muestra que un mayor porcentaje de entrenadores de básquetbol que menciona variables para la elección de los contenidos como el nivel de competencia y el encuadre del partido $[\chi 2(1, n=21)=6.30, p=.019]$.

\begin{tabular}{|c|c|c|c|}
\hline Nombre & & Fundamentado & Familias \\
\hline Voleibol & 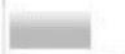 & 9 & 1. Deporte \\
\hline :̌: Básquetbol & Ea & 12 & 1. Deporte \\
\hline Rama Masculina & E & 14 & 2. Rama dirigida \\
\hline R: Rama Femenina & $E$ & 7 & 2. Rama dirigida \\
\hline u19 & $E$ & 10 & 3. Categoría dirigida \\
\hline \%15 & Eas & 11 & 3. Categoría dirigida \\
\hline u17 & E- & 11 & 3. Categoría dirigida \\
\hline 2.: Plan de juego & $E$ & 29 & I. Aspectos estratégicos \\
\hline Información del rival (scouting) & E & 17 & I. Aspectos estratégicos \\
\hline Apoyo a la autonomía & $+=$ & 9 & II. Aspectos psicológicos \\
\hline Regulación de la activación & E & 8 & II. Aspectos psicológicos \\
\hline Identidad de equipo / Comunicación grupal & - & 3 & II. Aspectos psicológicos \\
\hline Filosofía de afrontamiento de la competencia & 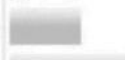 & 8 & II. Aspectos psicológicos \\
\hline Características del mensaje & & 15 & III. Aspectos comunicacionales \\
\hline Uso de recursos físicos / audiovisuales & 清 & 4 & III. Aspectos comunicacionales \\
\hline : Encuadre & Ear & 7 & IV. Variables que influyen en la ele... \\
\hline Nivel de competencia & Ea & 5 & IV. Variables que influyen en la ele... \\
\hline Contenidos especificos del entretiempo & E & 10 & V. Entretiempo \\
\hline
\end{tabular}

Figura 2. Frecuencia (fundamentación) de los códigos.

\section{DISCUSIÓN}

El objetivo del presente estudio fue explorar la percepción de los entrenadores acerca de los contenidos verbales que exponen durante las charlas precompetitivas y de entretiempo en partidos de voleibol y básquetbol de categoría formativa. El análisis de las entrevistas reveló que los entrenadores durante las charlas precompetitivas y de entretiempo tienen en cuenta cinco aspectos en relación con los contenidos que brindan: aspectos estratégicos, psicológicos, comunicacionales, variables que influyen en la elección de los contenidos y contenidos específicos de los entretiempos.

La primera de estas categorías (i.e. los aspectos estratégicos) refiere a la información del plan de juego tanto del propio equipo como del rival (scouting) mientras que los aspectos psicológicos reflejan las acciones realizadas por el entrenador que buscan 


\section{Raimundi, M. J.; Sarries, S.; Corti, J. F.; Celsi, I.}

producir un efecto en los aspectos personales de los deportistas, como la regulación de la activación, la manera de afrontar la competencia (el partido en sí), así como también en los aspectos sociales, como la identidad de equipo y la comunicación grupal.

Tabla 3

Prevalencia de las categorías centrales (familias de códigos) y de primer nivel (códigos)

\begin{tabular}{|c|c|c|c|c|c|c|c|c|}
\hline \multirow{2}{*}{ Categoría } & \multirow{2}{*}{$\begin{array}{c}\text { Entrenadores } \\
\text { que la } \\
\text { mencionan } \\
n(\%)\end{array}$} & \multicolumn{2}{|c|}{$\begin{array}{l}\text { En función del } \\
\text { deporte }\end{array}$} & \multicolumn{2}{|c|}{$\begin{array}{c}\text { En función de la rama } \\
\text { dirigida }\end{array}$} & \multicolumn{3}{|c|}{$\begin{array}{c}\text { En función de la categoría } \\
\text { dirigida }^{\mathrm{a}}\end{array}$} \\
\hline & & $\begin{array}{l}\text { Voleibol } \\
n(\%)\end{array}$ & $\begin{array}{l}\text { Básquetbol } \\
n(\%)\end{array}$ & $\begin{array}{l}\text { Masc. } \\
n(\%)\end{array}$ & $\begin{array}{l}\text { Fem. } \\
n(\%)\end{array}$ & $\begin{array}{c}\mathrm{u} 15 \\
n(\%)\end{array}$ & $\begin{array}{c}\mathrm{u} 17 \\
n(\%)\end{array}$ & $\begin{array}{c}\mathrm{u} 19 \\
n(\%)\end{array}$ \\
\hline I. Aspectos estratégicos & 20 (95.2) & $9(100)$ & 11 (91.7) & 13 (92.9) & $7(100)$ & $10(90.9)$ & $10(90.9)$ & $10(100)$ \\
\hline a. Plan de juego & $20(95.2)$ & $9(100)$ & $11(91.7)$ & $13(92.9)$ & $7(100)$ & $10(90.9)$ & $10(90.9)$ & $10(100)$ \\
\hline $\begin{array}{l}\text { b. Información sobre el rival } \\
\text { (Scouting) }\end{array}$ & 14 (66.7) & $6(66.7)$ & $8(66.7)$ & $9(64.3)$ & $5(71$ & $6(54.5)$ & $7(63.6)$ & $8(80)$ \\
\hline II. Aspectos psicológicos & $13(61.9)$ & $6(66.7)$ & $7(58.3)$ & $8(57.1)$ & 5 (71.4) & $6(54.5)$ & 7 (63.6) & $7(70)$ \\
\hline a. Regulación de la activación & $4(19)$ & $2(22.2)$ & $2(16.7)$ & $3(21.4)$ & $1(14.3)$ & $1(9.1)$ & $3(27.3)$ & $3(30)$ \\
\hline $\begin{array}{l}\text { b. Filosofía de afrontamiento de } \\
\text { la competencia }\end{array}$ & $5(23.8)$ & $2(22.2)$ & $3(25)$ & $4(28.6)$ & 3) & $3(27.3)$ & $1(9.1)$ & $2(20)$ \\
\hline c. Apoyo a la autonomía & $5(23.8)$ & $1(11.1)$ & $4(33.3)$ & $3(21.4)$ & $2(28.6)$ & $2(18.2)$ & $4(36.4)$ & $3(30)$ \\
\hline $\begin{array}{l}\text { d. Identidad de equipo / } \\
\text { Comunicación grupal }\end{array}$ & $3(14.3)$ & $1(11.1)$ & $2(16.7)$ & $2(14.3)$ & $1(14.3)$ & - & $2(18.2)$ & $3(30)$ \\
\hline III. Aspectos comunicacionales & $10(47.6)$ & $5(55.6)$ & $6(50)$ & $7(50)$ & $3(42.9)$ & $4(36.4)$ & $7(63.6)$ & $5(50)$ \\
\hline a. Características del mensaje & $9(42.9)$ & $5(55.6)$ & $4(33.3)$ & $6(42.9)$ & $3(42.9)$ & $4(36.4)$ & $7(63.6)$ & $4(40)$ \\
\hline $\begin{array}{l}\text { b. Uso de recursos físicos / } \\
\text { audiovisuales }\end{array}$ & $2(9.5)$ & - & $2(16.7)$ & $2(14.3)$ & - & - & - & $2(20)$ \\
\hline $\begin{array}{l}\text { IV. Variables que influyen en } \\
\text { la elección de contenidos }\end{array}$ & $6(28.6)$ & - & $6(50)$ & $5(35.7)$ & $1(14.3)$ & $3(27.3)$ & $3(27.3)$ & $3(30)$ \\
\hline a. Nivel de competencia & $4(19)$ & - & $4(33.3)$ & $3(21.4)$ & $1(14.3)$ & $3(27.3)$ & $2(18.2)$ & $1(10)$ \\
\hline b. Encuadre & $2(9.5)$ & - & $2(16.7)$ & $2(14.3)$ & - & - & $1(9.1)$ & $2(20)$ \\
\hline V. Entretiempo & $10(47.6)$ & $3(33.3)$ & $7(58.3)$ & $8(57.1)$ & $2(28.6)$ & $5(45.5)$ & 7 (63.6) & $5(50)$ \\
\hline $\begin{array}{l}\text { a. Contenidos específicos del } \\
\text { entretiempo }\end{array}$ & $10(47.6)$ & $3(33.3)$ & $7(58.3)$ & $8(57.1)$ & $2(28.6)$ & $5(45.5)$ & 7 (63.6) & $5(50)$ \\
\hline
\end{tabular}

aAlgunos entrenadores dirigen una única categoría, mientras que otros dirigen dos o tres. Para el cálculo de estos datos se contabilizó cada categoría de éstos últimos como entrenadores independientes.

A través de la observación de la conducta verbal, algunos autores (e.g. Crispim Santos, 2008; SánchezOliva et al., 2010) encontraron que los componentes tácticos y psicológicos son los más utilizados por los entrenadores de fútbol durante la competencia. Estos resultados coinciden con la percepción de los entrenadores sobre los contenidos incluidos, de acuerdo con los resultados del presente estudio. En lo que hace específicamente al básquetbol, el estudio realizado por Perea Villena (2008) se alinea con los resultados presentados, al señalar que los entrenadores durante la charla previa acuden mayormente a brindar información acerca del estilo de juego del rival, con la finalidad de facilitar una ventaja estratégica. Por lo tanto, la observación de la conducta verbal de los entrenadores coincidiría con la percepción que éstos tienen respecto de la importancia de los contenidos a desarrollar durante las competiciones.

De acuerdo con lo referido por los entrenadores, la siguiente categoría con mayor frecuencia alude a los aspectos comunicacionales, es decir, las características del mensaje y los recursos que los entrenadores priorizan al momento de comunicarse con sus deportistas durante charlas precompetitivas y de entretiempo. Uno de los aspectos emergentes dentro de esta categoría fue la utilización del "método o intervención sándwich” (R. E. Smith y Smoll, 2007) o del humor por parte del entrenador para optimizar la transmisión de información a sus deportistas. Resultados similares se hallaron en el trabajo de 


\section{Percepciones de entrenadores sobre charlas precompetitivas y entretiempos}

Mason et al. (2020), donde entrenadores de élite mencionaron la utilización de un "sándwich de feedback" que implica brindar una devolución negativa entre dos positivas.

El uso de la pizarra es otro de los recursos implementados con frecuencia por los entrenadores de ambos deportes. Lorenzo et al. (2013) plantean que existen diferencias entre las intervenciones del entrenador durante el tiempo de juego (período activo) y los momentos de pausa. En las primeras, los entrenadores tienden a utilizar el ánimo, feedback positivo, instrucciones físicas y técnicas, es decir, información que conlleva poco tiempo para que los deportistas puedan procesarla. Mientras que durante los tiempos muertos (períodos de pausa), los entrenadores emplean conductas verbales que conllevan mayor tiempo de procesamiento para el deportista como las estrategias tácticas, psicológicas y preguntas. Sin embargo, en el presente estudio los entrenadores perciben que también en los períodos de pausa (como las charlas previas y las de entretiempo) el tiempo para procesar la información es muy escaso $\mathrm{y}$, por ello, deben ser sintéticos y eficaces en la forma de comunicar, para no abrumar a sus jugadores. Uno de los entrenadores de voleibol de este estudio resaltó que no considera fructífera la transmisión de información compleja ni siquiera entre sets (i.e. momento de pausa de juego más extenso en los partidos de voleibol) y por ello puntualiza que suele trabajar los aspectos estratégicos en los entrenamientos previos a la competencia. Por lo tanto, a partir del análisis de las entrevistas, se observa que los entrenadores suelen tener presente que, al momento de comunicarse durante las competencias, tanto su habilidad para sintetizar la información en mensajes breves y eficaces, como la utilización de recursos que ayuden a sus jugadores a mantener la atención en la información brindada (como el humor o el "método sándwich"), resultan cruciales para que puedan asimilar esta nueva información y modificar su accionar en función del rendimiento.

Otra categoría encontrada refiere a que los contenidos seleccionados y desarrollados por los entrenadores se ven influenciados por aspectos contextuales como el nivel de competencia y su encuadre. Los entrevistados consideran que cuanto mayor es el nivel competitivo en el cual se está jugando, más estratégico y específico debe ser el contenido (del rival o del propio plan de juego). En consonancia con estos resultados, Mowat (2004) señala al nivel de la competición, los resultados previos contra un oponente concreto y el estado actual del partido como factores situacionales que pueden afectar la conducta de los entrenadores. Ahora bien, resulta interesante destacar que la incidencia de estas variables contextuales es reconocida sólo por algunos de los entrenadores del presente estudio (el 28.6\%, todos ellos entrenadores de básquetbol). Es posible pensar que, en muchos de los casos restantes, este efecto del contexto sobre los contenidos a transmitir se produzca de manera implícita. Mason (2020) muestra que lo que los entrenadores creen sobre el feedback que dan no siempre refleja cómo lo brindan y éste varía ampliamente según el contexto en el que se brinda. Esta variación entre contextos puede representar un área para mejorar la práctica del entrenador, pero también puede ser vista como una necesidad para adaptarse al entorno en el que se da esa conducta. Aún resta conocer con mayor profundidad las variables que pueden influir en ello, por lo tanto, puede ser interesante continuar investigando esta cuestión específica desde la percepción de los entrenadores.

En línea con las variables que pueden influir en la elección de los contenidos en las charlas previas y de entretiempo, también se encontró que un mayor número de entrenadores de básquetbol consideran el nivel de competencia, el momento de la temporada y la importancia del partido o la situación actual del equipo para seleccionar los contenidos a desarrollar. Resulta interesante enfatizar que los entrenadores no solo consideran un plan de juego fijo o metas establecidas con anterioridad, sino que se vuelven sensibles a las distintas necesidades que demanda la situación de partido y los jugadores de su equipo en momentos específicos de la temporada. En este sentido, la compleja tarea de los entrenadores requiere el desarrollo de habilidades específicas para prestar atención a las necesidades de los jugadores, a los cambios del contexto de la competencia, así como también para poder identificar y promover los intereses y preferencias de los jugadores, brindándoles mayor autonomía y animándonos a tomar el control de su propia participación (N. Smith et al., 2017; R. E. Smith y Smoll, 2007).

Por último, se señalan contenidos específicos del entretiempo, en los que se reflejan los ajustes, correcciones y redireccionamientos en función de cómo se esté dando el partido hasta el momento. Moreno Arroyo (2001) plantea que los entrenadores le conceden una gran importancia a los momentos de 


\section{Raimundi, M. J.; Sarries, S.; Corti, J. F.; Celsi, I.}

pausa del juego, ya que les permiten realizar modificaciones, así como también tener un contacto cercano y grupal con los jugadores, a fin de informar acerca de los aspectos claves que inciden en ese momento en el rendimiento del equipo y en el marcador. Esto guarda coherencia con lo reportado por los entrenadores de nuestro estudio, así como también con lo observado por Moreno Arroyo et al. (2002), quienes, a través de la observación de la conducta verbal, concluyen que durante los momentos de pausa del juego (intervalos entre sets), los entrenadores de voleibol incrementan la frecuencia de información táctica.

Los resultados de este trabajo no mostraron asociaciones significativas en la percepción de los entrenadores respecto a los contenidos a incluir en las charlas precompetitivas y de entretiempo en función de las categorías dirigidas (u15, u17 y u19). Los entrenadores no mostraron diferencias en la autopercepción de las conductas realizadas entre las distintas categorías de la etapa formativa. En estudios similares, se identificó que los entrenadores proporcionan mayor feedback específico positivo, negativo y correctivo en categorías formativas mientras que en las categorías senior acuden mayormente al ánimo, al sistema de juego del equipo y a las críticas a la organización (Guzmán Luján et al., 2014). Sin embargo, tal investigación no abordó si esta diferencia se encuentra ya, en menor grado, entre las distintas categorías formativas iniciales $y$ va aumentando conforme se acercan los jugadores a las categorías senior. Al estudiar las percepciones de las conductas de jugadores de voleibol y básquetbol, Fernández et al. (2018) encontraron que jugadores de la categoría formativa más alta (i.e., u19) perciben menor implicación en la tarea, mayor implicación en el ego y mayor estilo controlador por parte de sus entrenadores, mientras que las categorías formativas iniciales (u17, u15) perciben mayor implicación en la tarea y mayor apoyo social. Es decir, en las categorías formativas iniciales, los entrenadores se enfocan más en el aprendizaje, la mejora y el esfuerzo a la hora de comunicar, mientras que, en las categorías más grandes, los contenidos estarían más orientados a la competición con otros y a dar directivas y ejercer presión. Tomando estos resultados en consideración y siendo que en algunos casos los entrevistados hicieron referencia a esta disparidad en contenidos en función de la edad de sus entrenados, será pertinente indagar en mayor profundidad si efectivamente la autopercepción de las conductas que crean los entrenadores difiere cuando dirigen las diferentes categorías, a pesar de no haber alcanzado una asociación significativa en los resultados del presente estudio.

En este sentido, los resultados hallados en el presente estudio muestran, desde la propia perspectiva de los entrenadores la compleja tarea para organizar y brindar información durante las competiciones. Tarea de capital importancia que impacta en las experiencias deportivas de los jóvenes jugadores y jugadoras (Appleton et al., 2016; Lawrence y Kingston, 2007). Asimismo, de acuerdo con Mason et al. (2020), estos hallazgos ilustran la compleja y multifacética naturaleza de las autopercepciones de los entrenadores acerca de sus conductas en sus equipos deportivos.

Este trabajo presenta algunas limitaciones que deben ser señaladas. En primer lugar, el sesgo del género, dado que casi la totalidad de los participantes eran entrenadores y participó una sola entrenadora. Asimismo, el $67 \%$ de los entrenadores trabajaba en equipos de la rama masculina. Resta mucho por investigar acerca del rol moderador del género de los entrenadores y profesores y su interacción con el género de los equipos en las experiencias de los deportistas, dados los resultados aún inconsistentes entre sí (Castillo, Zamarripa et al., 2018) y dada la representación desigual que se sostiene en el ámbito del deporte respecto de la proporción de entrenadoras y entrenadores.

En segundo lugar, al considerar la perspectiva de los propios entrenadores, este estudio no valora lo que efectivamente realizan los entrenadores en sus charlas precompetitivas y de entretiempo sino la percepción que tienen sobre ello. Por lo tanto, para profundizar la comprensión de estos fenómenos, es importante continuar indagando con el fin de explorar la relación entre estas autopercepciones de los entrenadores, la percepción de los deportistas acerca de estos contenidos y los perfiles conductuales que estos llevan a cabo durante la competencia (Guzmán Luján et al., 2014). Diversos estudios en contextos deportivos y de actividad física muestran discrepancias entre la conducta y las percepciones de los entrenadores o profesores y los deportistas o practicantes (Alves Franco et al., 2013; N. Smith et al., 2017) y muestran la inexactitud de los entrenadores cuando reflexionan acerca de las conductas que tienen en sus entornos deportivos (Mason et al., 2020). Sin embargo, al considerar todas las fuentes de información, los 


\section{Percepciones de entrenadores sobre charlas precompetitivas y entretiempos}

modelos orientados a predecir la motivación de los deportistas se vuelven más robustos (N. Smith et al., 2016). Asimismo, se ha mostrado que el feedback que los entrenadores brindan a sus deportistas no equivale al feedback que éstos reciben, por lo tanto, será importante explorar qué métodos pueden evaluar cómo reciben información los deportistas acerca de ese feedback que dan los entrenadores (Mason, 2020). En esta línea, el desarrollo o la adaptación de instrumentos destinados a registrar la conducta verbal del entrenador en los tiempos de pausa (i.e. charlas previas, entretiempos, tiempos muertos) representaría un avance para responder a la diversidad $\mathrm{y}$ singularidad de los casos (Anguera et al., 2007). Asimismo, facilitaría la obtención de información para contribuir a la mejora de las habilidades de los entrenadores durante estos momentos deportivos específicos (Hastie, 1999). Con ello, podría continuar profundizándose el estudio de la percepción de los entrenadores acerca de su propia conducta verbal, no sólo durante las competencias, sino también en entrenamientos y durante las charlas posteriores a los partidos, posibilitando un entendimiento más global de los procesos de comunicación entre ellos y sus jugadores. Conocer los criterios de evaluación de rendimiento y el valor que se le otorga a los resultados en esas circunstancias deportivas, articulado con las conductas efectivamente realizadas y la percepción de sus jugadores, permitirá un análisis pormenorizado de los efectos de las creencias del entrenador respecto de sus conductas verbales y sobre las experiencias de los deportistas.

Finalmente, resultaría pertinente evaluar cómo administran y qué contenidos incluyen en sus comunicaciones los entrenadores de otros deportes, principalmente de deportes individuales, dado que la frecuencia y duración de las pausas en las que pueden intervenir suele diferir en gran medida respecto de los deportes colectivos. Los estudios respecto de las creencias y autopercepciones de los entrenadores acerca del feedback que brindan a sus deportistas aún es escasa y se encuentra en desarrollo (Mason et al., 2020; Roberts et al., 2019). Por ello, se espera continuar en la complementariedad cualitativocuantitativo que promueven los métodos mixtos para potenciar las aplicaciones prácticas de los trabajos realizados (Anguera y Hernández-Mendo, 2016).

\section{APLICACIONES PRÁCTICAS}

Este trabajo puede contribuir al autoconocimiento de los entrenadores respecto a sus intervenciones en las charlas precompetitivas y de entretiempo, como momentos determinados de interacción con sus jugadores en deportes específicos como el voleibol y el básquetbol. Conocer qué contenidos se priorizan en estos deportes y qué estrategias se utilizan para transmitirlos, como así también las variables a tener en cuenta a la hora de decidir esa forma y contenidos a transmitir, aporta a los entrenadores y profesores en el conocimiento e información sobre sus propias intervenciones.

Asimismo, el trabajo de los profesionales de la Psicología en el contexto deportivo podrá tener en cuenta estas concepciones de los entrenadores a la hora de comunicarse con sus deportistas y sus equipos, para poder brindar estrategias comunicacionales eficaces, que permitan mejorar el rendimiento de los entrenadores como líderes y orientadores de sus jugadores. Asimismo, la enseñanza de estrategias psicológicas, como aquellas que buscan la regulación emocional de los entrenadores durante la competencia, ayudándolos a ponerse en el lugar de sus deportistas y favoreciendo su participación a través del feedback significativo, puede constituir un puntapié inicial para tal objetivo. Al optimizar las herramientas para comunicar que utilizan los entrenadores, será posible incidir de manera indirecta en las experiencias deportivas de los deportistas, facilitando un mayor disfrute y rendimiento en la actividad (Borrueco et al., 2020; Vives-Ribó y Rabassa, 2020).

\section{AGRADECIMIENTOS}

A los/as entrenadores/as que nos prestaron sus voces y permitieron la realización del estudio y al equipo de investigación que realizó la recolección de datos.

\section{REFERENCIAS}

Adie, J., Duda, J. L., y Ntoumanis, N. (2012). Perceived Coach Autonomy Support, basic need satisfaction and the well-and ill-being of elite youth soccer players: A longitudinal investigation. Psychology of Sport and Exercise, 13(1), 51-59. https://doi.org/10.1016/j.psychsport.2011.07.00 $\underline{8}$

Alves Franco, S. C., Da Costa Simões, V. A., Castañer, M., De Jesus Fernandes Rodrigues, J., y Anguera, M. T. (2013). La conducta de los 


\section{Raimundi, M. J.; Sarries, S.; Corti, J. F.; Celsi, I.}

instructores de fitness: Triangulación entre la percepción de los practicantes, auto-percepción de los instructores y conducta observada. Revista de Psicología del Deporte, 22(2), 321-329. https://www.redalyc.org/pdf/2351/2351280580 27.pdf

Anguera, M. T. (1990). Metodología observacional. En J. Arnau, M. T. Anguera y J. Gómez-Benito (Eds.), Metodología de la investigación en ciencias del comportamiento (pp. 125-236). Universidad de Murcia.

Anguera, M. T., Blanco-Villaseñor, Á., HernándezMendo, A., y Losada López, J. L. (2011). Diseños observacionales: Ajuste y aplicación en psicología del deporte. Cuadernos de Psicología del Deporte, 11(2), 63-76. https://revistas.um.es/cpd/article/view/133241

Anguera, M. T., y Blanco-Villaseñor, Á. (2003). Registro y codificación del comportamiento deportivo. En A. Hernández-Mendo (Ed.), Psicología del Deporte (Vol. II) Metodología (pp. 6-34). EFDeportes.com.

Anguera, M. T., y Hernández-Mendo, A. (2013). La metodología observacional en el ámbito del deporte. E-balonmano.com: Revista de Ciencias del Deporte, 9(3), 135-160. http://diposit.ub.edu/dspace/bitstream/2445/108 477/1/629241.pdf

Anguera, M. T., y Hernández-Mendo, A. (2016). Avances en estudios observacionales de ciencias del deporte desde los mixed methods. Cuadernos de Psicología del Deporte, 16(1), 17-30. https://revistas.um.es/cpd/article/view/254261

Anguera-Argilaga, M. T., Magnusson, M. y Jonsson, G. (2007). Instrumentos no estandar: planteamiento, desarrollo y posibilidades. Avances en medición, 5(1), 63-82. https://dialnet.unirioja.es/servlet/articulo?codig $\underline{\mathrm{o}=2981059}$

Appleton, P. R., Ntoumanis, N., Quested, E., Viladrich, C. y Duda, J. L. (2016). Initial validation of the coach-created Empowering and Disempowering Motivational Climate Questionnaire (EDMCQ-C). Psychology of Sport and Exercise, 22, 53-65. https://doi.org/10.1016/j.psychsport.2015.05.00 $\underline{8}$

Ato, M., López, J., y Benavente, A. (2013). Un sistema de clasificación de los diseños de investigación en psicología Introducción Un marco conceptual para la investigación. Anales de Psicología, 29(3), 1038 -

1059. https://doi.org/10.6018/analesps.29.3.178 $\underline{511}$

Balagué Serre, N., Torrents Martín, C., Pol Cabanellas, R., y Seirul-lo Vargas, F. (2014). Entrenamiento integrado. Principios dinámicos y aplicaciones. Apunts Educación Física y Deportes, (116), 60-68.

https://doi.org/10.5672/apunts.2014-

0983.es.(2014/2).116.06

Balaguer, I., Castillo, I., y Duda, J. L. (2008). Apoyo a la autonomía, satisfacción de las necesidades, motivación y bienestar en deportistas de competición: Un análisis de la teoría de la autodeterminación. Revista de Psicología del Deporte, 17(1), 123-139. https://www.redalyc.org/pdf/2351/2351192460 02.pdf

Borrueco, M., Ramis, Y., Pallarès, S., y Cruz, J. (2020). Aplicación del modelo de las 5Cs para la formación de entrenadores: Un ejemplo de práctica basada en la evidencia. Revista de Psicología Aplicada al Deporte y al Ejercicio Físico, 5(1),

8. https://doi.org/10.5093/rpadef2020a3

Cajina-Guedeat, M., y Reyes-Bossio, M. (2021). Experiencia emocional subjetiva en deportistas extremos: estudio cualitativo. Cuadernos de Psicología del Deporte, 21(1), 1-17. https://revistas.um.es/cpd/article/view/418141

Calpe-Gómez, V., Guzmán Luján, J. F., y Grijalbo Santamaría, C. (2013). Relationship between score and coaches' verbal behaviour. Journal of Human Sport and Exercise, 8(3), 728-737. https://doi.org/10.4100/jhse.2013.8.Proc3.19

Castillo, I., Adell, F. L., y Alvarez, O. (2018a). Relationships between personal values and leadership behaviors in basketball coaches. Frontiers in Psychology, 9. https://doi.org/10.3389/fpsyg.2018.01661

Castillo, I., Zamarripa, J., Tomás, I., Álvarez, O., Delgado, M., y Marentes, M. (2018b). Teacher psychological needs support, gender and autonomous motivation in Physical Education students. 23rd. Annual Congress of the European College of Sport Sciences (ECSS).

Cohen, J. (1960). A coefficient of agreement for nominal scales. Educational and Psychological 


\section{Percepciones de entrenadores sobre charlas precompetitivas y entretiempos}

Measurement, 20(1), 37-46. https://doi.org/10.1177/001316446002000104

Creswell, J. W. (2013). Research design: Qualitative, quantitative, and mixed methods approaches. SAGE publications.

Crispim Santos, A. (2008). Análisis de la instrucción del entrenador de fútbol. Comparación entre el instrucción de preparación y de competición. Fitness \& Performance Journal, 7(2), 112-122. https://doi.org/10.3900/fpj.7.2.112.s

Curran, T., Hill, A. P., Hall, H. K., y Jowett, G. E. (2015). Relationships between the coach-created motivational climate and athlete engagement in youth sport. Journal of Sport and Exercise Psychology, 37(2), 193-198. https://doi.org/10.1123/jsep.2014-0203

Fernández, A., García-Arabehety, M., Celsi, I., Tourn, S., y Raimundi, M. J. (2018). Clima motivacional creado por los entrenadores: diferencias en la percepción de deportistas adolescentes de Buenos Aires (Argentina) en función del género, deporte y categoría. VII Congreso Iberoamericano de Psicología del Deporte.

Guzmán Luján, J. F., Calpe-Gómez, V., Grijalbo Santamaría, C., y Imfeld Burkhard, F. (2014). Una observación sistemática de las conductas verbales de los entrenadores en función de las acciones de juego competitivas. Revista de Psicología del Deporte, 23(2), 301-307. https://www.redalyc.org/articulo.oa?id=235131 674010

Guzmán Luján, J. F., y Calpe-Gómez, V. (2012). Preliminary study of coach verbal behaviour according to game actions. Journal of Human Sport and Exercise, 7(2), 376-382. https://doi.org/10.4100/jhse.2012.72.04

Hastie, P. (1999). An instrument for recording coaches' comments and instructions during time-outs. Journal of Sport Behavior, 22(4), 467-478.

https://doi.org/https://psycnet.apa.org/record/19 99-15471-001

Hernández-Sampieri, R. (2018). Metodología de la investigación: las rutas cuantitativa, cualitativa y mixta. McGraw-Hill Interamericana.

Landis, J. R., y Koch, G. G. (1977). The Measurement of Observer Agreement for Categorical Data. Biometrics, 33(1), 159-174. https://doi.org/10.2307/2529310
Lapresa Ajamil, D., Otero, A., Arana, J., Álvarez, I., y Anguera, M. T. (2021). Concordancia consensuada en metodología observacional: efectos del tamaño del grupo en el tiempo y la calidad del registro. Cuadernos de Psicología del Deporte, 21(2), 47-58. https://doi.org/10.6018/cpd.467701

Lawrence, G., y Kingston, K. (2007). Skill Acquisition for Coaches. En R. L. Jones, M. Hughes, y K. Kingston (Eds.), An Introduction to Sports Coaching. Routledge.

Lorenzo, J., Navarro, R., Rivilla, J., y Lorenzo, A. (2013). The analysis of the basketball coach speech during the moments of game and pause in relation to the performance in competition. Revista de Psicología del Deporte, 22(1), 227230.

https://www.redalyc.org/pdf/2351/2351275520 27.pdf

Luque, M. M., Reigal-Garrido, R. E., Baro, J. P. M., Sánchez, V. M., y Mendo, A. H. (2019). Estilo de interacción del entrenador, clima motivacional percibido y satisfacción de las necesidades psicológicas básicas en futbolistas jóvenes. Cuadernos De Psicología Del Deporte, 19(3), 79-89. https://doi.org/10.6018/cpd.372381

Marcelo, C., y Gallego-Domínguez, C. G. (2018). “¿Quién soy yo como maestro?" Construcción de la identidad profesional en docentes principiantes. En I. Cantón, y M. Tardif (Eds.), Identidad profesional docente, 45-56. Narcea.

Marques, M., Nonohay, R., Koller, S., Gauer, G., y Cruz, J. (2015). El estilo de comunicación del entrenador y la percepción del clima motivacional generado por los entrenadores y compañeros. Cuadernos de Psicología Del Deporte, 15(2), 47-54. https://doi.org/10.4321/S157884232015000200006

Mason, R. J. (2020). Examining the knowledge, use, and reception of verbal coach feedback across high performance sport environments. [Tesis doctoral, The University of Melbourne]. http://hdl.handle.net/11343/249852

Mason, R. J., Farrow, D., y Hattie, J. A. (2020). Sports Coaches' Knowledge and Beliefs About the Provision, Reception, and Evaluation of Verbal Feedback. Frontiers in Psychology, 11, 2424. https://doi.org/10.3389/fpsyg.2020.571552 


\section{Raimundi, M. J.; Sarries, S.; Corti, J. F.; Celsi, I.}

Maxwell, J. (2012). Qualitative research design: An interactive approach (3era ed.). SAGE Publications.

Molina, M. F., y Schmidt, V. (2020). Relationship between Family Functioning and Possible Selves in Adolescents from Argentina. The Journal of Genetic Psychology, 181(5), 319-335. https://doi.org/10.1080/00221325.2020.175889 $\underline{8}$

Morales-Sánchez, V., Pérez-López, R., Reigal, R. E., y Hernández-Mendo, A. (2020). Mixed-Methods Analysis of Emotional Quality in Sports Organizations: Facial Expressions of Child Users of Sports Services as Data. Frontiers in Psychology, 11 . https://doi.org/10.3389/fpsyg.2020.01199

More, K., y Franks, I. M. (2004). Measuring coaching effectiveness. En M. Hughes y I. M. Franks (Eds.), Notational Analysis of Sport: Systems for Better Coaching and Performance in Sport. Routledge.

Moreno Arroyo, M. (2001). Análisis y optimización de la conducta verbal del entrenador de voleibol durante la dirección de equipo en competición. [Tesis doctoral, Universidad de Extremadura]. http://dehesa.unex.es/handle/10662/583

Moreno Arroyo, M., Santos, J., Ramos Mondéjar, L., Sanz, D., Fuentes García, J., y Villar Álvarez, F. (2002). Aplicación de un sistema de codificación para el análisis de contenido de la conducta verbal del entrenador de voleibol. European Journal of Human Movement, 9, 119-140. https://recyt.fecyt.es/index.php/ejhm/article/vie $\mathrm{w} / 56185 / 34060$

Mowat, T. J. (2004). Arousal and behaviour of coaches during competition. [Tesis doctoral, Victoria University of Technology]. http://vuir.vu.edu.au/15646/

Perea Villena, P. (2008). Análisis descriptivo de la conducta verbal del entrenador de base en balonmano. EfDeportes Revista Digital, 119. https://www.efdeportes.com/efd119/conductaverbal-del-entrenador-de-base-enbalonmano.htm

Pérez-López, R., Morales-Sánchez, V., Teresa Anguera, M. T., y Hernández-Mendo, A. (2015). Evaluación de la calidad total en servicios municipales deportivos orientados a la población infantil: Aportaciones desde el análisis cualitativo con ATLAS.ti. Cuadernos de
Psicologia del Deporte, 15(1), 143-150. https://doi.org/10.4321/S157884232015000100014

Roberts, A. H., Greenwood, D. A., Stanley, M., Humberstone, C., Iredale, F., y Raynor, A. (2019). Coach knowledge in talent identification: A systematic review and metasynthesis. Journal of Science and Medicine in Sport, 22(10), $1163-$

1172. https://doi.org/10.1016/j.jsams.2019.05.0 $\underline{08}$

Sánchez-Oliva, D., Sánchez Miguel, P. A., Amado Alonso, D., Leo Marcos, F. M., y García Calvo, T. (2010). Análisis de la conducta verbal del entrenador de fútbol en función de su formación federativa y del periodo del partido en categorías inferiores. Retos. Nuevas tendencias en Educación Física, Deporte y Recreación, 18, 24-28.

https://doi.org/10.47197/retos.v0i18.34646

Schmidt, V., Marconi, A., Messoulam, N., Maglio, A., Molina, F., y González, M. A. (2008). La comunicación entre padres e hijos desde la percepción adolescente. Una aproximación etnopsicológica. Revista de Psicología Social Aplicada, 17(1), 1-23. https://etci.com.ar/descargas/Caracterizacionde-la-comunicacion.pdf

Smith, M., y Cushion, C. J. (2006). An investigation of the in-game behaviours of professional, toplevel youth soccer coaches. Journal of Sports Sciences, 24(4), 355-366. https://doi.org/10.1080/02640410500131944

Smith, N., Quested, E., Appleton, P. R., y Duda, J. L. (2016). A review of observational instruments to assess the motivational environment in sport and physical education settings. International Review of Sport and Exercise Psychology, 9(1), 134-159.

https://doi.org/10.1080/1750984X.2015.113233 4

Smith, N., Quested, E., Appleton, P. R., y Duda, J. L. (2017). Observing the coach-created motivational environment across training and competition in youth sport. Journal of Sports Sciences, 35(2), 149-158. https://doi.org/10.1080/02640414.2016.115971 $\underline{4}$

Smith, R. E., y Smoll, F. L. (2007). Social-cognitive approach to coaching behaviours. En S. Jowett y 


\section{Percepciones de entrenadores sobre charlas precompetitivas y entretiempos}

D. Lavallee (Eds.), Social Psychology in Sport. Human

Kinetics. https://doi.org/10.5040/978149259587 $\underline{8}$

Strauss, A. y Corbin, J. (1990). Basics of qualitative research: Grounded Theory procedures and techniques. SAGE Publications.
Strauss, A. y Corbin, J. (1997). Grounded Theory in Practice. SAGE Publications.

Vives-Ribó, J., y Rabassa, N. (2020). Intervención sobre la comunicación del entrenador para aumentar la confianza de los deportistas. Revista de Psicología Aplicada al Deporte y al Ejercicio Físico, 5(1), 6. https://doi.org/10.5093/rpadef2020a1 